\title{
Présentation. Pour une « cartographie » de la critique architecturale
}

Hélène Jannière

\section{(2) OpenEdition}

Journals

Édition électronique

URL : http://journals.openedition.org/crau/290

DOI : $10.4000 /$ crau.290

ISSN : 2547-5746

Éditeur

Éditions du patrimoine

Édition imprimée

Date de publication : 1 décembre 2009

Pagination : 14-19

ISBN : 978-2-85822-944-4

ISSN : 1296-4077

Référence électronique

Hélène Jannière, «Présentation. Pour une « cartographie » de la critique architecturale », Les Cahiers de la recherche architecturale et urbaine [En ligne], 24/25 | 2009, mis en ligne le 01 septembre 2017, consulté le 21 avril 2019. URL : http://journals.openedition.org/crau/290 ; DOI : 10.4000/crau.290 


\section{Présentation Pour une " cartographie " de la critique architecturale}

Durant les quinze dernières années, ouvrages, articles et événements publics sur la critique architecturale (son rôle et son rapport au projet $d^{\prime}$ 'architecture) se sont multipliés ${ }^{1}$. Parallèlement, dans la décennie 1990, critiques, historiens et architectes s'interrogeaient sur la « topographie » incertaine des tendances architecturales. Progressivement, les débats (ceux qui étaient focalisés sur la postmodernité architecturale comme ceux qui étaient suscités par la déconstruction en architecture) laissaient place à des positionnements architecturaux plus individualisés, propres à chaque architecte ou même à chaque projet $d^{\prime}$ 'architecture ${ }^{2}$. Mais au moment où de nombreux observateurs européens (critiques ou historiens de l'architecture) soulignaient cette nouvelle réalité des doctrines architecturales, allant même jusqu'à faire état d'une "crise », intervenaient d'importants changements de ligne éditoriale au sein de plusieurs grandes revues européennes, dont Casabella ${ }^{3}$. Sans nécessairement imputer à cette reconfiguration des débats architecturaux un renouveau de l'intérêt pour la critique, on peut souligner la concomitance des deux phénomènes. Loin d'être inédite, cette mise en relation de la crise présumée de la production architecturale et de la crise présumée de la critique

1. Par exemple, le NethCa a organisé en avril 2003 à Bruxelles deux journées d'étude sur les instruments de la critique, "Critical Tools ». En 2002, Archilab (FRAC-Centre, Orléans) a animé un débat intitulé de manière quelque peu erronée "Premier symposium international de la critique d'architecture », le sujet n'étant pas inédit pour un rassemblement international d'architectes et de critiques. Plus récemment à Koweit
City, les 6 et 7 décembre 2005, le Comité International des Critiques d'Architecture (CICA) a proposé un congrès "Architectural Journalism and Criticism " (en partenariat avec l'Aga Khan Award for Architecture, la Kuwait Society of Engineers et la Kuwait League of Architects). Du 23 au 25 avril 2009, I'université de Trondheim, en Norvège, a organisé un colloque "Architectural Research and Architectural Criticism », explorant les apports mutuels entre 
architecturale s'est exprimée à de nombreuses reprises au cours du XX $X^{e}$ siècle, les concepts de crise et de critique étant par ailleurs étymologiquement et historiquement liés ${ }^{4}$.

Symptôme d'une crise, la critique est souvent réputée être elle-même en crise, voire menacée de disparition. Ainsi François Burckhardt regrettait-il en 1997 la fin de la " grande critique » due selon ses termes à la disparition d'une génération, celle des Giulio Carlo Argan, Reyner Banham, Manfredo Tafuri ${ }^{5}$. Aujourd'hui, avec ce dossier des Cahiers, une nouvelle contribution collective sur la critique architecturale veut aller au-delà de ces constats, au-delà de tentatives de typologie de la critique ${ }^{6}$. Elle veut dépasser la discussion déjà ancienne sur les complexités de la critique architecturale et sur les difficultés à la cerner en tant que pratique autonome et en tant que forme d'écrit spécifique. L'approche de ce dossier propose au contraire de décrypter cet objet singulier qu'est la critique architecturale. Discours aux multiples registres, elle couvre un large spectre d'écrits, depuis le discours d'évaluation héritier du " jugement» proche des définitions canoniques de la critique d'art, jusqu'aux essais théoriques issus de la critical theory et du post-structuralisme. On ne peut définir «la » critique architecturale comme pratique ou genre unique de discours sur l'architecture car elle est forcément située dans l'espace et dans le temps.

Au sein d'une « cartographie » de la critique architecturale, au moins deux conceptions distinctes de la critique se dessinent: I'une la rattache aux investigations théoriques sur l'architecture, I'autre la rattache aux pratiques journalistiques sur l'architecture dans la presse spécialisée ou généraliste. En France, le mot « critique » renvoie majoritairement à cette seconde conception (la critique comme pratique, attachée à une économie, à un marché, à des acteurs, etc.). Dans d'autres pays, la critique est à dominante historique ou philosophique ; elle entre parfois en relation directe avec l'élaboration d'un champ théorique architectural autonome. Le mot " critique ", dans le seul domaine de l'architecture, recouvre selon les contextes culturels des acceptions distinctes. Partir de l'une ou de l'autre de ces définitions a des conséquences sur les modes d'appréhension de la critique, sur sa constitution en objet de recherche, comme le montrent les quelques exemples qui suivent.

En Italie, la plupart des recherches récentes sur la critique architecturale - objet d'un constant débat depuis les années 1950 - adoptent un point de vue historique, en étroite relation avec le développement de "I'histoire de l'histoire de l'architecture » (notamment du Mouvement moderne) et la publication des monographies sur des historiens critiques (on peut lire par exemple les travaux de Carlo Olmo et son équipe à I'Université polytechnique de Turin, ainsi que les ouvrages de Renato De Fusco $^{7}$ ). II convient par ailleurs de souligner l'importance de tous ces écrits, qui reviennent sur les définitions de la " critique opératoire » telles que données par Manfredo Tafuri, dès 1968. Beaucoup d'entre eux explorent les relations entre critique et projet (on peut citer notamment les articles sur la critique dans Casabella depuis les années 1980, ainsi que le volume de Luca Monica paru en 2002 sur La Critica operativa ${ }^{8}$ qui reprend le contenu des débats entre Giulio Carlo Argan, Bruno Zevi et d'autres critiques italiens sur ce sujet). Dès 1999, le recherche et critique (Nordic Association for Architectural Research, Faculty of Architecture and Fine Art, Norvegian University of Science and Technology). Quant aux revues d'architecture, elles s'interrogent régulièrement sur la critique en tant que discipline et en tant que pratique.

2. Les années 1980 se caractérisaient par une transition entre la « crise de la confrontation entre les tendances, une fois absorbée dans le marché actuel des images, et une nouvelle recherche qui prend ses distances de cette dispute, en se proposant de résoudre le problème de manière individuelle à l'occasion de chaque projet » (Leonardo Benevolo, «Benevolo risponde a Rykwert: sui rapporti fra critica e architettura », Casabella, n 522, mai 1986, p. 38).

3. La fin de la direction de Vittorio Gregotti qui, la décennie précédente, avait publié une série d'articles sur la critique, ses relations à l'histoire, aux théories et au projet.
4. Voir Reinhardt Koselleck, Le règne de la critique, Paris, Les Éditions de Minuit, 1979 (édition originale Kritik und Krise. Ein Beitrag zur Pathogenese der bürgerlichen Welt, Fribourg, K. Alber, 1959).

5. François Burckhardt, in Dietmar Steiner, "Architecture and Media: the Future of the Architectural Magazines. Symposium », Domus, nº 790 (février 1997), pp. 51-58. 
numéro 21 de la revue internationale d'architecture Zodiac dirigée par Guido Canella (avec des articles de Carlo Olmo, Jean-Louis Cohen, Ignasi de Solá-Morales, Michela Rosso...) prenait acte de la fin de la critique " opératoire » et engagée et, dans cette perspective, abordait l'actualité des relations entre critique et histoire en s'interrogeant sur leur devenir.

En Allemagne, l'objet de recherche nommé " critique ", moins associé à l'histoire que dans la plupart des études italiennes, est présent dans l'enseignement de I'architecture. Plusieurs séminaires lui sont consacrés 9 . Les publications récentes issues de ces activités académiques couvrent un large spectre de définitions, de pratiques et de types d'écrits, depuis le « journalisme architectural » (Architekturjournalismus) jusqu'aux théories de l'architecture ${ }^{10}$. La critique y semble majoritairement perçue comme un discours spécifique, voire autonome, ayant ses propres règles, en étroite relation avec les publications architecturales et le monde de la presse.

Aux États-Unis enfin, l'expression architectural criticism recouvre deux activités distinctes. Elle renvoie d'une part à la tradition d'une critique architecturale de qualité, virulente et militante, établie depuis plusieurs décennies dans la grande presse (Lewis Mumford, Ada Louise Huxtable...). Elle désigne d'autre part une activité spéculative dans le champ des théories architecturales. Ainsi, la théorie critique et l'histoire critique appliquées à l'architecture dans les universités américaines depuis la fin des années 1970 ont été diffusées par des revues telles que Oppositions, October ou Assemblage. Elles ont été également véhiculées par les conférences Any (dont une édition a été consacrée à la critique). On peut citer en outre les écrits de Kenneth Frampton, de Joan Ockman ou de Mary McLeod sur les relations entre critique, théorie et histoire (à propos de la revue Perspecta ${ }^{11}$ mais également à propos de la théorie critique dans le volume Architecture, Criticism, Ideology publié en $1988^{12}$ ).

En France, plusieurs publications ont depuis 1995 jalonné le débat. Parmi les plus marquantes, le premier numéro du Visiteur (automne 1995) a relancé l'intérêt pour la critique et interrogé cette pratique avec les contributions de Bernard Huet, Jean-Louis Cohen, Maurice Culot, Jacques Lucan, François Chaslin, à la suite d'un cycle de conférences organisé par Sébastien Marot en 1991 à la Société française des architectes ${ }^{13}$. Plus récemment, un ouvrage collectif paru aux Éditions de La Villette (La critique architecturale. Questions, frontières, desseins ${ }^{14}$ ) rassemble des études sociologiques, historiques ou philosophiques sur le sujet, des témoignages de critiques français et étrangers contemporains. À ces écrits récents s'adjoignent dans cet ouvrage des textes republiés, comme celui de Michel Ragon, critique d'art français ayant au seuil des années 1960 consacré son énergie à la critique de l'architecture, Thomas Creighton, ancien rédacteur en chef de Progressive Architecture ou Julius Posener, tous parus dans le numéro de L'Architecture d'aujourd'hui de $1964^{15}$.

Dans ce dossier, il a été convenu d'explorer un premier thème transversal: identifier les différentes conjonctures qui permettent à cette critique architecturale d'advenir, d'exister et de se développer de manière impliquée et située. Ainsi Kenneth Frampton se saisit-il de l'exemple de la scène architecturale britannique de
6. Dans le domaine de l'art et de la littérature, de tels constats ont été dépassés et de nouvelles interrogations se sont fait jour sur les fonctions actuelles de la critique. Pour une approche générale des nouvelles questions, voir par exemple la revue Tracés Revue de sciences humaines, $n^{\circ} 13$, "Où en est la critique? », 2007, outre les nombreuses publications sur la critique d'art contemporaine.
7. Renato De Fusco, L'Idea di architettura. Storia della critica da Viollet-le-Duc a Persico, Milan, Comunità, 1964, et R. De Fusco, Cettina Lenza, Nuove idee di architettura. La critica da Rogers a Jencks, Milan, Etas, 1991

8. Luca Monica, La Critica operativa e I'architettura, Milan, Éditions Unicopli, 2002.

9. Par exemple à l'université de Cottbus (séminaire dirigé par Christian Gänshirt), mais aussi dans les universités de Kassel, Brauschweig ou encore Leipzig.

10. Ulrich Conrads, Eduard Führ et Christian Gänshirt (dir.), Zur Sprache bringen. Kritik der Architekturkritik, Münster, Waxmann Verlag, 2003

11. Robert A. M. Stern, Peggy Deamer et Alan Plattus (dir.), Re-reading Perspecta: The First Fifty Years of the Yale Architectural Journal, Cambridge, Mass. / Londres, MIT Press, 2004. 
I'après-guerre jusqu'aux années 1970 pour examiner les relations entre la critique entendue comme discours (celle des historiens Rudolf Wittkower et Colin Rowe comme celle des critiques Alan Colquhoun et Reyner Banham) et la réception critique des productions de l'architecture. À partir du cas de la réception critique en Grande-Bretagne des travaux de Le Corbusier, il pose la question du projet comme contribution critique au débat architectural. II interroge également les frontières entre critique et réception de l'architecture.

Autre milieu, autre "projet critique ", d'une tout autre nature celui-ci : Pierre-Alain Croset et Michele Bonino évoquent le projet de "réalisme critique " de Vittorio Gregotti dans Casabella de 1982 à 1996. Ils montrent d'une certaine manière « la critique au travail » en décryptant la démarche de Gregotti, entouré d'une équipe internationale. Ils analysent le rapport entre ce projet critique, la structure en rubriques et la forme graphique de la revue elle-même.

Un entretien avec Ulrich Conrads, rédacteur en chef de la revue Bauwelt de 1957 à 1988, fondateur de Stadtbauwelt en 1964 et de la revue théorique Daidalos en 1981, évoque la critique architecturale berlinoise des années 1950 à 1980. Pour Carsten Krohn qui commente cet entretien, la critique exercée pendant plusieurs décennies par Conrads ne se limite pas à l'appréhension esthétique d'un objet architectural, ni même à l'élaboration de critères architecturaux pour l'analyse de telle ou telle production. Lorsqu'elle concerne l'aménagement urbain, ce qui est fréquemment le cas, cette critique architecturale est à ses yeux indissociable de l'engagement politique.
Dans le contexte new-yorkais des années 1930 à aujourd'hui, l'essai de Suzanne Stephens de la revue Architectural Record interroge le fossé grandissant qui sépare la critique publiée dans la presse nationale et la critique publiée par les universités américaines depuis les années 1970. Elle retrace le parcours intellectuel et «médiatique » de critiques tels que Lewis Mumford, Ada Louise Huxtable ou Herbert Muschamp.

Avec ce large tour d'horizon, la succession de ces contributions ne parcourt pas uniquement des situations géographiques et des contextes culturels différents. Elle reflète aussi diverses conceptions de la critique tout en éclairant le débat « post critical/ critical» développé aux États-Unis depuis le début de cette décennie.

Le deuxième thème transversal qui structure ce dossier des Cahiers est l'orientation théorique de la critique, symbolisée par le terme d' 'école critique " que I'on peut définir à partir de la relation entre la production d'un discours critique et le positionnement théorique (à l'intérieur et à l'extérieur du champ architectural). On peut penser à I'" école critique " de Manfredo Tafuri (marxiste et post-marxiste) telle qu'elle a été développée à l'Istituto Universitario di Architettura di Venezia, telle qu'elle a été élaborée collectivement à travers les écrits de Tafuri, Francesco Dal Co, Giorgio Ciucci, Marco de Michelis, Mario Manieri-Elia, et telle qu'elle est aujour$d^{\prime}$ 'hui amplement commentée ${ }^{16}$. Sur le sujet plus contemporain des débats que suscite dans la dernière décennie I'oppo-sition " post critical/critical », Manuel Martín Hernández examine de son côté les limites entre critique et théorie. Dans le contexte historique qui articule la période dite "moderne » et le mouvement qualifié de
12. Joan Ockman, Deborah Bercke et Mary McLeod (dir.), Architecture Criticism Ideology, Princeton, Princeton Architectural Press, 1985.

13. La revue Le Visiteur a été créée par la Société française des architectes en 1995 à l'initiative de Sébastien Marot; pour être tout à fait complet, on précisera que la revue Le Visiteur, interrompue en 2003 après dix numéros, est à nouveau publiée par la Société française des architectes avec un tout autre projet éditorial (www.levisiteur.com). On soulignera également l'existence d'une nouvelle revue bisannuelle, Criticat, publiée depuis janvier 2008 par l'association éponyme (www.criticat.fr)
14. Agnès Deboulet, Rainier Hoddé et André Sauvage (dir.), La critique architecturale. Questions, frontières, desseins, Paris, Éditions de La Villette, 2008.

15. L'Architecture d'aujourd'hui, $n^{\circ} 117$, novembre 1964 
"postmoderne » en architecture, Jacques Lucan explore quant à lui la question du langage de la critique, notamment lorsque celui-ci donne lieu aux interprétations de la critique européenne dans les théories architecturales outre-Atlantique. Enfin, dans la continuité de cet appel pour une « cartographie » de la critique architecturale, il conviendra de s'interroger sur la possibilité de constituer la critique en objet de recherche (avec son chantier épistémologique, ses périmètres, ses terrains et ses méthodes), un préalable peut-être nécessaire à la poursuite d'une investigation scientifique sur un sujet très polémique.

16. Parmi les plus récentes des études, voir Andrew Leach, Manfredo Tafuri. Choosing History, Gand, A\&S Books, 2007; Marco Biraghi, Progetto di crisi. Manfredo Tafuri e l'architettura contemporanea, Milan, Marinotti, 2005. Voir en outre les nombreux articles sur l'historien, ainsi que le numéro spécial de Casabella, no 619-620, janvierfévrier 1995. 


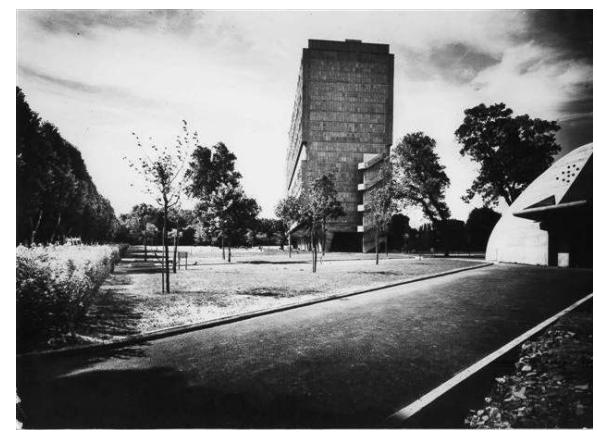

Unité d'habitation

de Marseille. Le Corbusier

(1945-1952) : espace vert.

FLC L1(14)29.

(c) FLC/ADAGP. 